\title{
Aerosol in selected laboratories at Faculty of Mechanical Engineering, Opole University of Technology
}

\author{
Tomasz Olszowski ${ }^{1, *}$ \\ ${ }^{1}$ Department of Thermal Engineering and Industrial Facilities, Opole University of Technology, Mikołajczyka 5, 45-271 Opole, \\ Poland
}

\begin{abstract}
The paper contains the results of a study into mass concentration of the dispersed aerosol fraction with the aerodynamic diameter of up to 2.5 and 10 micrometers. The study was conducted during classes with students participating in them in two laboratories located at Faculty of Mechanical Engineering, Opole University of Technology as well as outdoor outside the building. It was demonstrated that the values of the mass concentration of $\mathrm{PM}_{2.5}$ and $\mathrm{PM}_{10}$ measured in the laboratories differ considerably from the levels measured in the ambient air in the outdoor areas surrounding the faculty building. It was concluded that the diversity of $\mathrm{PM}_{2.5} / \mathrm{PM}_{10}$ ratio was greater in the laboratories. Direct correlation was not established between the concentrations of the particular PM fractions in the two investigated environments. It was demonstrated that there is a statistically significant relation between the concentration of $\mathrm{PM}_{2.5}$ and $\mathrm{PM}_{10}$ and the number of people present in the laboratory. The conducted cluster analysis led to the detection of the existence of dominant structures determining air quality parameters. For the analyzed case, endogenic factors are responsible for the aerosanitary condition. The study demonstrated that the evaluation of air quality needs to be performed individually for the specific rooms.
\end{abstract}

\section{Introduction}

Epidemiological studies have shown that there is a strong correlation between the air pollutants concentration and increase the prevalence of respiratory diseases and finally mortality of people $[1,2]$. People spend $85-90 \%$ of their time in enclosed spaces, including $40 \%$ in buildings with public access [3]. For this reason, it is important to pay attention to the parameters of air which they used to breathe. One of the parameters of air quality is associated with the mass concentration of particulate matter suspended in the air.

Over the years, it was taken that indoor air quality is directly relative to the parameters of the external air penetrating the buildings. Nevertheless, around the turn of last century, it was observed that the concentration of aerosols inside the buildings is often considerably higher in comparison to the quality of external air infiltrating the area [4].

The recent studies indicate that the air quality indoors is relative to both the factors associated with the external environment as well as endogenic factors. The studies reported in [5] deal with the characteristics of the factors affecting the quality of air inside buildings, which were found to include air pollution, microclimate conditions, level of air ionization, exploitation conditions of the buildings as well as their design. The factors deciding about the quality of the inside environment include the ones associated with the presence as well as animal and human activity indoors. The activities inside buildings can considerably affect not only on the level of the concentration of classical aerosol particles as well as the level of bioaerosols [6-8]. Air quality is also considerably influenced by the effect resulting from the existence of ventilation and air conditioning systems. The concentration of aerosol particles is also dependent on the season. In the moderate climate, higher aerosol concentrations are registered during winter [9].

This paper focuses on the verification of the level of mass concentration of $\mathrm{PM}_{2.5}$ and $\mathrm{PM}_{10}$ in the selected laboratory rooms of a university during the classes with students participating in them. It also aims to determine the relation between the particulate matter concentration and the parameters which characterize the quality of air outdoors.

\section{Materials and methods}

\subsection{Measurement sites}

The study was conducted in the laboratories in the conditions when students participated in the classes. The laboratories are situated at the Faculty of Mechanical Engineering, Opole University of Technology $\left(50^{\circ} 40^{\prime} 59.9^{\prime \prime} \mathrm{N} ; 17^{\mathrm{O}} 56^{\prime} 42.62 " \mathrm{E}\right)$. It was ensured that the rooms selected for the testing were similar in size and layout. For the purpose of minimizing the impact of direct insolation on the potential air movement indoors, the two selected laboratories were located adjacent and

Corresponding author: t.olszowski@po.opole.p1 
protected from the direct exposition to sunlight. The first of them, Atmospheric Pollution Control laboratory (APC) has a cubic capacity of $98 \mathrm{~m}^{3}$, whereas the other one, Thermodynamic laboratory (TD) comprises $118 \mathrm{~m}^{3}$. The rooms are used periodically (only during classes) and are regularly cleaned up. The rooms comprise the same resources in terms of the material, design etc. The air flow resulting from leakages through drafty windows and doors is characterized by similar infiltration parameters. Both rooms receive ventilation air through outdated gravitational ventilation.

In addition, with the purpose of determining the potential impact of the external conditions on the air quality indoors, the measurements of the dedicated quantities were also performed outdoors. The equipment, including aerosol aspirator and temperature sensor was exposed outside the rooms on an observation platform situated 3 meters away from the windows in the horizontal plane.

\subsection{Measurements procedure and results analysis}

The mass concentration of the aerosol fractions of up to 2.5 and 10 micrometers in size was measured concurrently by application of Environmental DustTrak Aerosol Monitor (Models 8335 DRX TSI ${ }^{\circledR}$ ). Every day, prior to the measurements, the apparaus was calibrated. It was done with the purpose of setting the value of the calibration coefficient to match to the current parameters of the indoor and outdoor air. Concurrently, prior to the specific measurements, the appatus was set to zero. The flow of air through the measurement cells of the apparatus was always equal to $1\left[\mathrm{dm}^{3} \cdot \mathrm{min}^{-1}\right]$ during the observations.

The aspiration of $\mathrm{PM}_{2.5}$ and $\mathrm{PM}_{10}$ was performed for the time constant of 10 minutes and the registration constant of 10 seconds. The data acquisition and storage occurred automatically. The registration involved 960 independent observations indoors and 96 outdoors.

Throughout the aerosol aspiration, data regarding outdoor and indoor air temperature was registered. In addition, the number of persons present in class was also recorded.

The raw data was verified by the Shapiro-Wilk test with the purpose of determination of the distribution of the measurement data. The data did not demonstrate normal distribution, hence, the analysis of the registered data was performed by the use of a non-parametric test. The comparison of the results applied Mann-Whitney U test.

Another verification of the dependencies applied the cluster analysis (CA) [10]. It was done with the aim of obtaining a uniform group of the tested objects, thus facilitating the identification of the principal characteristics and structure of the analyzed data, consequently enabling the researcher to classify the standard objects.

As a result of the objective analysis of the data cluster, it will be possible to establish the mutual relations between the elements forming a given set. This is particularly relevant in the aspect of modeling processes occurring in the ambient air. The relations between the parameters characterizing the ambient air are particularly important during the estimation of the parameters regarding probability distribution, offering the further assessment of the variability of air quality in rooms.

The analysis applied all investigated variables. The data was rescaled on the basis of the algorithm (1), which reduced the impact of the absolute value of a given variable and enabled all variables to be limited within the range $\{0 ; 1\}$. The analysis applied the procedure of agglomeration - by determining the measure of the distance between the objects (Euclidean distance).

$$
X^{\prime}=\frac{X-X_{\min }}{X_{\max }-X_{\min }}
$$

\section{Results and discussion}

Table 1 summarizes the data on the number of observations of selected parameters of air and data on the use of classrooms. The results suggest that the physical parameters and the number of students present in the laboratores were almost identical in both rooms. A comparison undertaken by means of the Wilcoxon test demonstrated a lack of the differences both in terms of the aerosanitary conditions and number of students present in class (p-value 0.18 accompanied by a materiality level $\mathrm{a}=0.05$ ). This, in turn, implies that the further study should adopt observations with regard to the environment that are discussed without classification of the data according to the location of the rooms.

Table 1. Selected variables collection.

\begin{tabular}{|c|c|c|c|c|c|}
\hline Site & $\begin{array}{c}\text { No of } \\
\text { observ. }\end{array}$ & $\begin{array}{c}\mathbf{P M}_{\mathbf{2 . 5}} \\
{\left[\boldsymbol{\mu} \mathbf{g} \cdot \mathbf{m}^{-3}\right]}\end{array}$ & $\begin{array}{c}\mathbf{P M}_{\mathbf{1 0}} \\
{\left[\boldsymbol{\mu} \mathbf{g} \cdot \mathbf{m}^{-3}\right]}\end{array}$ & $\begin{array}{c}\mathbf{T} \\
{\left[{ }^{\mathbf{O}} \mathbf{C}\right]}\end{array}$ & $\begin{array}{c}\text { No of } \\
\text { people }\end{array}$ \\
\hline APC & 640 & & & & \\
Min & & 32.5 & 40.0 & 19.1 & 3.00 \\
Max & & 179 & 215 & 23.3 & 14.0 \\
AVG & & 64.5 & 89.5 & 22.1 & 8.80 \\
SD & & 34.6 & 53.8 & 1.69 & 3.90 \\
\hline TD & 320 & & & & \\
Min & & 31.2 & 39.9 & 19.0 & 4.00 \\
Max & & 186 & 219 & 23.1 & 15.0 \\
AVG & & 63.5 & 89.9 & 22.0 & 9.00 \\
SD & & 35.8 & 51.4 & 1.71 & 3.95 \\
\hline O & 96 & & & & \\
Min & & 30.1 & 42,0 & -4.00 & $\mathrm{n} / \mathrm{a}$ \\
Max & & 95.3 & 151 & 19.0 & $\mathrm{n} / \mathrm{a}$ \\
AVG & & 49.6 & 69.6 & 8.80 & $\mathrm{n} / \mathrm{a}$ \\
SD & & 29.9 & 24.5 & 8.09 & $\mathrm{n} / \mathrm{a}$ \\
\hline
\end{tabular}

Fig. 1 demonstrates the results of the mass concentration of aerosol with the fractions of up to a 2.5 and 10 micrometers in size. Fig. $1 \mathrm{~A}$ presents the results of the mass concentration recorded during the measurements in the indoor (I) and outdoor air (O) during the episodes of the unstable weather (temperature $16-18{ }^{\mathrm{O}} \mathrm{C}$, light wind, good dispersion of pollution). Fig. 
1B presents the results accompaying the occurrence of smog episodes in the urban area (temperature inversion, $\mathrm{T}$ in the range from -4 to $-1{ }^{\mathrm{O}} \mathrm{C}$, windless weather). In both cases, the values of the mass concentrations of $\mathrm{PM}_{2.5}$ and $\mathrm{PM}_{10}$ established in the rooms signifcantly differ from the levels that were determined in the outdoor air in the vicinity of the faculty building.
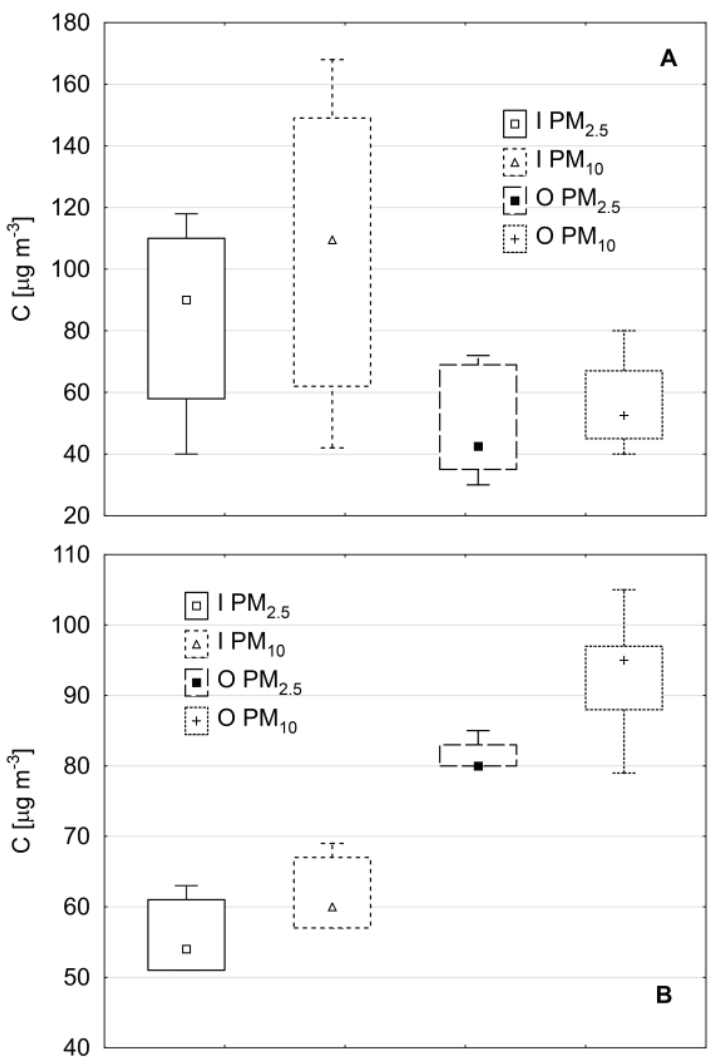

Fig. 1. Mass concentration of $\mathrm{PM}_{2.5}$ and $\mathrm{PM}_{10}$ for different weather conditions. Boxes show the range between the $25^{\text {th }}$ and $75^{\text {th }}$ percentiles. The whiskers extend from the edge of the box to the $5^{\text {th }}$ and $95^{\text {th }}$ percentiles of the data. The squares inside indicate median values.

The results of the Wilcoxon test unequivocally confirm the differences. For the adopted materiality level of $\alpha=0.05, p$-value was equal to 0.004 , which clearly confirms the existance of the statistically significant differences between the concentrations in the two environments. It is noteworthy that the concentrations of aerosols indoors do not seem to be relative to the outdoor parameters. The indoor temperature was virtually constant (coefficient of variability $\mathrm{Cv}$ was lower than $0.1 \%$ ). The outdoor temperature was characterized by a greater variability $(\mathrm{CV} \approx 0.92 \%)$. Within the range of similar temperatures (results derived from 600 observations (I) and $60(\mathrm{O})$, when the difference between the indoor temperature (I T) and the outdoor temperature (O T) was around 20\%), we observed from 1.5 to 4 times higher concentrations of the particular fractions in the rooms. This demonstrates the impact of the local sources of pollutants as well as inadequate ventilation. Concurrently, the faulty ventilation aided in the maintenance of higher standards of the indoor air quality during the smog episodes, when the measured concentrations of $\mathrm{PM}_{2.5}$ and $\mathrm{PM}_{10}$ were, respectively, 25 and $35\left[\mu \mathrm{g} \mathrm{m}^{-3}\right]$ lower in the indoor air compared to the outdoor air. Unfortunately, after a comparison with the 24-hour values, the $\mathrm{PM}_{10}$ levels recorded in the laboratories were found to exceed the admissible levels.

The comparison of the outdoor concentrations of $\mathrm{PM}_{10}$ with the results gathered in the laboratories [11] demonstrates that the indoor air quality is more adverse. The mass concentrations of the aerosols in the laboratories is nearly $40\left[\mu \mathrm{g} \cdot \mathrm{m}^{-3}\right]$ higher than the mass concentration of the particles with the same fraction. The ratio of the dispersed phase in the aerosol can be assessed to be negligible. The considerable differences in the $\mathrm{PM}_{10}$ values are attributable equally to the poor ventilation, as well as to the character of the space utilization during the classes. The activity in the laboratories, in contrast to lecture rooms, is associated with a considerable degree of physical movement of the students in class. In turn, this results in the escalation of the $\mathrm{PM}_{\mathrm{X}}$ emission from the clothes and skin, which is accompanied by a permanent phenomenon of the resuspension of solid particles from the surface. Consequently, this contributes to the enrichment of the air with the solid particles.

The study did not involve the measurements of total suspended particles (TSP). The ratio of $\mathrm{PM}_{10}$ in the TSP is in the range from 0.4 to 0.8 [12]. Under the assumption that this ratio is low in the laboratories, we can assume that during the cold season, and in the conditions marked by the insufficient quality of ventilation and air filtration, aerosanitary conditions in the laboratories are extremely adverse. In accordance with data provided by DECOS [13], a safe level of total suspended particles is equal to $200 \mu \mathrm{g} \cdot \mathrm{m}^{-3}$ in the consideration of the maximum anticipated ratio of the fine particle fraction. For the lowest ratio of $\mathrm{PM}_{10}$, the value of TSP is exceeded.

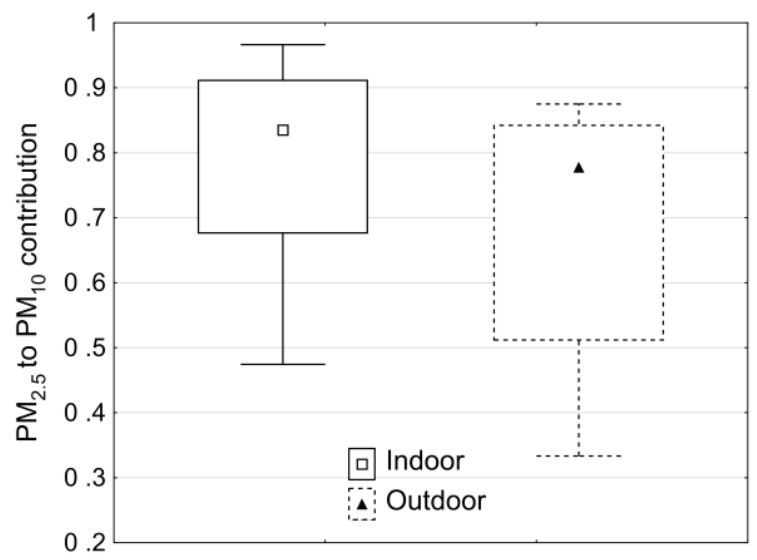

Fig. 2. Ratio of $\mathrm{PM}_{2.5}$ in $\mathrm{PM}_{10}$.

Fig. 2 presents the ranges of the ratios of the respirable fraction in $\mathrm{PM}_{10}$ in the two environments. A basic statistical analysis yieds that a greater diversity of the ratio of $\mathrm{PM}_{2.5}$ in $\mathrm{PM}_{10}$ is noted in the rooms. The $\mathrm{Cv}$ value was equal to $0.11 \%$ for $(\mathrm{I})$ and $0.05 \%$ for $(\mathrm{O})$. The statement regarding the greater diversity of the ratio of $\mathrm{PM}_{2.5}$ in $\mathrm{PM}_{10}$ noted in the rooms means that the variety and the number of the emission sources in the rooms have a considerable impact on the aerosol concentration. 
Only for the case of the outdoor aerosol, the sources of emission are not that much diversified. The difference that is clearly visible in the figure is also confirmed by the results of the Wilcoxon test ( $p$-value $=0.039$, for $\alpha=0.05)$.

Separately for the internal and external environment, there is a heavy dependence between the mass concentration of $\mathrm{PM}_{2.5}$ and $\mathrm{PM}_{10}$. The Speraman's rho- was equal to 0.92 and 0.98 for (I) and (O), respectively.

Table 2 contains the results for the Spearman's rank correlation between the registered parameters.

Table 2. Spearman correlation coefficient values for selected parameters.

\begin{tabular}{|c|c|c|c|}
\hline $\begin{array}{c}\text { I PM }_{2,5} \text { to } \\
\mathrm{O} \mathrm{PM}_{2,5}\end{array}$ & $\begin{array}{c}\text { I } \mathrm{PM}_{10} \text { to } \\
\mathrm{OPM}_{10}\end{array}$ & 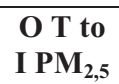 & $\begin{array}{l}\text { O T to } \\
\text { I PM }_{10}\end{array}$ \\
\hline-0.188 & -0.298 & 0.205 & 0.237 \\
\hline $\begin{array}{l}\text { I T to I } \\
\text { PM }_{2,5}\end{array}$ & $\begin{array}{c}\text { I T to I } \\
\text { PM }_{10}\end{array}$ & $\begin{array}{l}\text { I P to } \\
\text { I } \mathbf{P M}_{2,5}\end{array}$ & $\begin{array}{l}\text { I P to } \\
\text { I } P_{10}\end{array}$ \\
\hline 0.374 & 0.456 & 0.678 & 0.728 \\
\hline
\end{tabular}

On the basis of the Guilford scale [14], we can conclude about the lack of a correlation between the concentrations of the particular particle fractions in both environments. The relation between the indoor concentration of $\mathrm{PM}_{2.5}$ and $\mathrm{PM}_{10}$ and the temperature (and therefore also weather) does not seem to be statistically material. A weak, yet significant, positive correlation was established for the indoor temperature and mass concentration of aerosol in the laboratories. A statistically significant and strong dependence $\left(\mathrm{PM}_{2.5}\right)$ and certain relation $\left(\mathrm{PM}_{10}\right)$ regarding the mutual impact of the two parameters was established for the mass concentration of $\mathrm{PM}_{2.5}$ and $\mathrm{PM}_{10}$ and the number of students present in the laboratory.

A graphical representation of the cluster analysis is presented in Fig. 3. The conducted classification offered the possibility to classify the groups of the analyzed variables.

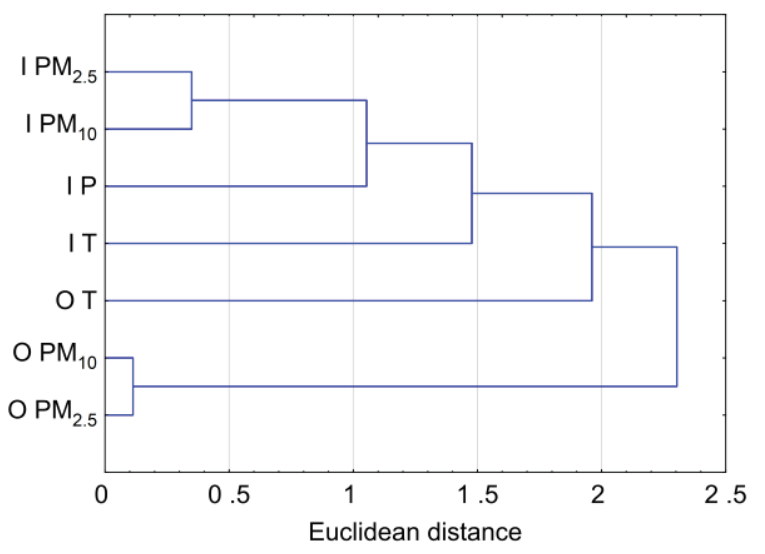

Fig. 3. Dendrogram for the verified objects.

Fig. 3 demonstrates that the most homogenous group is formed by the variables characterizing the parameters measured in the laboratory. The computed Euclidean distance between I $\mathrm{PM}_{2.5}$ and $\mathrm{I} \mathrm{PM}_{10}$ was equal to 0.35 , whereas between I PMx and I P and I T, it was 1.07 and 1.57, respectively. For the remaining parameters, the distances exceeded the indicative value of 2 , which clearly demonstrates the divergences with regard to the homogeneity of the parameter types (I) and (O). The performed analysis confirms that the values of the parameters determined for the outdoor environment are considerably distinct from the ones that were established for the laboratory environment. This result can confirm the impact of the particular parameters on the value of the mass concentration of $\mathrm{PM}_{2.5}$ and $\mathrm{PM}_{10}$ in the laboratories.

\section{Conclusions}

In the analyzed case, the aerosanitary conditions are primarily determined by the endogenic factors. Undoubtedly the results are affected by the physical containment of the laboratory space resulting from the poor ventilation in them. The considerable degree of limitation of air exchange with the ambient air result in the maintenance of qualitative parameters in the rooms only during the smog episodes. In general, during the heating season, the aerosanitary conditions have to be considered as unsatisfactory. The study also demonstrated that the assessment of indoor air quality should be performed individually for every case. Concurrently, it is known that the modernization of the ventilation systems in the examined laboratories is an urgent necessity.

\section{References}

1. ECA. 22 (2000)

2. US EPA. EPA/600/P-99/002bF; (2004)

3. L. Morawska, C.R. He, G. Johnson, H., E. Uhde, G. Ayoko, Environ Sci Technol. 43 (2009)

4. W.J. Fisk, D. Faulkner, D. Sullivan, M.J. Mendell, Aerosol Sci Tech. 32(6) (2000)

5. H. Fromme, J. Diemer, S. Dietrich, J. Cyrys, J. Heinrich, W. Lang, M. Kiranoglu, D. Twardella, Atmos Environ. 42 (2008)

6. A. Afshari, U. Matson, L.E. Ekberg, Indoor Air. 15(2) (2005)

7. T. Hussein, K. Hameri, M.S.A. Heikkinen, M. Kulmala M. Atmos Environ. 39 (2005)

8. H. Guo, L. Morawska, C.R. He, D. Gilbert, Atmos Environ. 42 (2008)

9. L. Morawska, C.R. He, G. Johnson, H. Guo, E. Uhde, G. Ayoko, Environ. Sci Technol. 43 (2009)

10. P.J. Rousseeuw, J. Comput App Math 20 (1998)

11. M. Lenart, T. Olszowski, Ecol Chem Eng A 23(2) (2016)

12. C. Alves, T. Nunes, J. Silva, M. Duarte, Aerosol Air Quality Res 13 (2013)

13. B. Kromhourt, H.J. Boleij, Ann Occup Hyg. 39 (1995)

14. J.P. Guilford, Psychometric methods (New York, McGraw-Hill, 1954) 